\title{
Using tuberculosis surveillance data for informed programmatic decision-making
}

Nobuyuki Nishikioria and Fukushi Morishita ${ }^{a}$

$\mathrm{P}$ ublic health surveillance, through the ongoing and systematic collection, analysis, interpretation and dissemination of health information, plays a critical role in informed decision-making and appropriate public health action. ${ }^{1}$ Different surveillance systems are required to meet disease-specific public health objectives, and some systems have been expanded through the collection of risk factor, behaviour and health practice information.

Tuberculosis (TB) control programmes are widely known as successful public health programmes that effectively collect and use surveillance data in a standardized manner at both the global and national levels. Surveillance was one of the five core components in the original World Health Organization (WHO) Framework for Effective Tuberculosis Control (the WHO DOTS strategy) established in 1994. The strategy recommended a case-based registry and cohort monitoring of treatment outcomes using highly standardized, paper-based recording and reporting forms. Once an individual was recorded on the TB register, heath workers monitored and recorded the person's treatment until completion. Thus the recording and reporting system of TB programmes, as well as being a surveillance tool, also served as a patient support tool.

In addition to monitoring individual patient outcomes, there are several other uses of surveillance data for informed programmatic decision-making (Box 1). These include measurement of disease burden, detection of potential outbreaks, definition of high-risk groups and monitoring and evaluation of programme performance. ${ }^{2}$ Further analysis of surveillance data can help identify programmatic gaps, prioritize interventions and research topics and allocate resources for subsequent action.

\section{Assessing disease burden and epidemiological trends}

TB surveillance data have been the globally recognized, fundamental data source for assessing the

\section{Box 1. Uses of TB surveillance data for programmatic decision-making}

- To assess disease burden and epidemiological trends

- To monitor unusual occurrences and phenomena for potential response actions

- To evaluate programmatic impacts

- To identify high risk, vulnerable or underserved populations for programmatic targeting

- To investigate epidemiological processes, especially in the context of contact investigation using genotyping technology

disease burden and epidemiological trends of $\mathrm{TB}^{3}$ WHO's policy for measuring TB disease burden emphasizes the importance of quality surveillance towards the ultimate goal of measuring TB incidence directly from TB notifications. ${ }^{4}$ Annually, WHO collects TB surveillance data from more than 200 countries and publishes the epidemiological situation of TB at the country, regional and global levels. WHO also promotes in-depth analysis of national surveillance data through various workshops and trainings.

\section{Monitoring unusual occurrences and phenomena for potential response actions}

Another fundamental function of a surveillance system is to detect unusual occurrences of a disease including outbreaks, such as the outbreaks of multidrugresistant TB in the Federated States of Micronesia. ${ }^{5}$ TB surveillance data can also be combined with other data sources, such as genotyping and geospatial data, to determine possible transmission chains of TB cases and to identify clusters. ${ }^{6}$ In the same study, routinely reported data were used to formulate an outbreak prediction algorithm for the automatic generation of outbreak alerts, identification of high-risk clusters and potential candidates for intensified contact investigation.

\footnotetext{
a World Health Organization Regional Office for the Western Pacific, Manila, Philippines doi: 10.5365/wpsar.2013.4.1.007
} 


\section{Evaluating of programmatic impact}

Data from a reliable TB surveillance system with reasonable coverage can be used to evaluate TB programmes. This is a great advantage for TB programmes, as the evaluation components of pilot or programmatic operational research can be assessed using routinely collected TB surveillance data. Three examples of such evaluation are published in this issue of WPSAR. By using TB surveillance data, Shimouchi et al. demonstrated that appropriate patient support significantly improved treatment outcome and reduced the drug resistance rate in Osaka city between 2001 and $2008 .^{7}$ Similarly, Ngamvithayapong-Yanai et al. used TB surveillance data to assess the programmatic impact of social mobilization activities in Thailand. ${ }^{8}$ By comparing TB surveillance data in project and non-project districts, Lu et al. demonstrated improved treatment outcome among internal migrants with TB who received subsidiary support in Shanghai. ${ }^{9}$

\section{Identifying high-risk, vulnerable or underserved populations}

In-depth analysis of surveillance data can reveal high-risk groups who are vulnerable to the disease. Yan $F$ et al. analysed TB surveillance data and reported a substantial burden of TB among prisoners in Mongolia. ${ }^{10}$ Improved TB management in the prison sector achieved significant reduction in TB notification over a decade. ${ }^{10}$ Foreign-born individuals are among the most vulnerable populations in many countries with a low TB incidence. ${ }^{11}$ The epidemiology of TB among foreign-born persons is often investigated, especially in countries with high immigration and low TB burden with the use of surveillance data. ${ }^{11}$ In this issue of WPSAR, Uchimura et al. analysed Japan's routine TB surveillance data to examine different high-risk groups including those with human immunodeficiency virus (HIV) infection, diabetes mellitus patients, contact cases, homeless people, foreigners, health care workers and the elderly. They found that the risk groups in Japan were quite different from other high-income countries with a low TB burden. ${ }^{12}$

Analysis of TB surveillance data can be further enhanced by linking with socioeconomic variables from other data sources. In this issue of WPSAR, Wong et al. combined TB surveillance data with data from poverty surveys and demographic health surveys in Cambodia to assess the relationship between TB diagnosis and poverty. ${ }^{13}$ The recent advancement in geographical information system technologies provides unlimited potential for geographical linkage between surveillance and other data, including census data. While recognizing methodological limitations (such as ecological fallacy), this type of analysis improves our understanding of the role of social determinants in TB epidemiology, potentially facilitates targeted interventions and should be explored further.

Since the 1994 WHO DOTS strategy, TB surveillance systems have evolved due to social, demographic, technological and epidemiological changes. Standardized recording and reporting forms have been revised and capture newly identified programme components such as TB/HIV activities and the management of multidrug-resistant TB. The introduction of electronic and/or web-based surveillance systems have greatly improved the accessibility and timeliness of TB surveillance data, ${ }^{2}$ further contributing to the widespread utilization of TB surveillance data in parallel with the development of innovative analysis approaches. However, as for all surveillance systems, TB surveillance data carry inherent limitations when it comes to scientific rigorousness, and TB notifications may not be considered a direct measure or close proxy of TB incidence. Continued efforts are needed to improve the quality of surveillance data, and careful assessment of the accuracy, completeness and comprehensiveness is required when interpreting the result of the analysis.

Despite these limitations, TB surveillance continues to serve as the fundamental data source for TB programme management and decision-making. Basic skills and knowledge on methodologies to analyse surveillance data should be widely disseminated through practical hands-on capacity building activities at the national and subnational levels. Technical advances in infectious disease epidemiology, geo-spatial analysis and molecular epidemiology should be actively applied for analysing TB surveillance data to improve our understanding of TB epidemiology. The sharing of such experiences among countries will further stimulate ideas and encourage a culture of evidence-based programme management and policy formulation. In this regard, WPSAR is one venue for this communication. 


\section{References:}

1. Nsubuga $P$ et al. Public Health Surveillance: A Tool for Targeting and Monitoring Interventions. In: Jamison DT et al, eds. Disease Control Priorities in Developing Countries, 2nd edition. Washington, DC, World Bank, 2006. pmid:21250309

2. Nadol $P$ et al. Electronic tuberculosis surveillance systems: a tool for managing today's TB programs. The International Journal of Tuberculosis and Lung Disease, 2008, 12(Suppl 1):8-16. pmid: 18302816

3. Dye $\mathrm{C}$ et al. Consensus statement. Global burden of tuberculosis: estimated incidence, prevalence, and mortality by country. WHO Global Surveillance and Monitoring Project. JAMA: the Journal of the American Medical Association, 1999, 282:677-686. doi:10.1001/jama.282.7.677 pmid:10517722

4. Bierrenbach A, Floyd K. TB impact measurement: policy and recommendations for how to assess the epidemiological burden of TB and the impact of TB control. Geneva, World Health Organization, 2009 (http://whqlibdoc.who.int/publications/ 2009/9789241598828_eng.pdf, accessed 1 March 2013).

5. Centers for Disease Control and Prevention (CDC). Two simultaneous outbreaks of multidrug-resistant tuberculosis-Federated States of Micronesia, 2007-2009. MMWR. Morbidity and Mortality Weekly Report, 2009, 58:253-256. pmid:19300407

6. Althomsons SP et al. Using routinely reported tuberculosis genotyping and surveillance data to predict tuberculosis outbreaks. PLoS ONE, 2012, 7:e48754. doi:10.1371/journal. pone.0048754 pmid:23144956

7. Shimouchi A et al. Strengthened tuberculosis control programme and trend of multidrug resistant tuberculosis rate in Osaka City,
Japan. Western Pacific Surveillance and Response Journal, 2013, 4(1). doi:10.5365/wpsar.2012.3.4.015

8. Ngamvithayapong-Yanai $\mathrm{J}$ et al. Engaging women volunteers of high socioeconomic status in supporting socioeconomically disadvantaged tuberculosis patients in Chiang Rai, Thailand. Western Pacific Surveillance and Response Journal, 2013, 4(1). doi:10.5365/wpsar.2012.3.4.013

9. Lu H et al. Do transportation subsidies and living allowances improve tuberculosis control outcomes among internal migrants in urban Shanghai, China? Western Pacific Surveillance and Response Journal, 2013, 4(1). doi:10.5365/wpsar.2013.4.1.003

10. Yanjindulam $P$ et al. Reduction of tuberculosis burden among prisoners in Mongolia: review of case notification, 2001-2010. [Notes from the field]. The International Journal of Tuberculosis and Lung Disease, 2012, 16:327-329. doi:10.5588/ ijtld.11.0251 pmid:22640445

11. Alvarez GG et al. A comparative examination of tuberculosis immigration medical screening programs from selected countries with high immigration and low tuberculosis incidence rates. BMC Infectious Diseases, 2011, 11:3. doi:10.1186/14712334-11-3 pmid:21205318

12. Uchimura K. Characteristics and treatment outcomes of tuberculosis cases by risk groups, Japan, 2007-2010. Western Pacific Surveillance and Response Journal, 2013, 4(1). doi:10.5365/wpsar.2012.3.016

13. Wong $A$ et al. The association between household poverty rate and tuberculosis case notification rate in Cambodia, 2010. Western Pacific Surveillance and Response Journal, 2013, 4(1). doi:10.5365/wpsar.2013.4.1.002 